\title{
Co-DAS: Development and Evaluation of Co-Driver Assistance System Concepts to Reduce Passenger Discomfort
}

\author{
Sandra Ittner (ORCID: https://orcid.org/0000-0003-2568-0789) ${ }^{1}$, Dominik Mühlbacher ${ }^{1}$, Mark Vollrath \\ (ORCID: https://orcid.org/0000-0001-8508-8934), Thomas H. Weisswange (ORCID: \\ https://orcid.org/0000-0003-2119-6965) ${ }^{2}$ \\ 1 WIVW GmbH, Veitshöchheim, Germany; ${ }^{2}$ Honda Research Institute Europe GmbH, Offenbach, \\ Germany, ${ }^{3}$ Technische Universität Braunschweig, Braunschweig, Germany
}

\begin{abstract}
The front seat passenger is often neglected when developing support systems for cars. There exist few examples of systems that provide information or interaction possibilities specifically to those passengers. Previous research indicated that the passive role of the passenger can frequently lead to a feeling of discomfort, potentially caused by missing information and missing control with respect to the driving situation. This paper proposes a variety of prototypical passenger assistance systems that target different aspects of the cognitive processes which could cause the feeling of discomfort. In a simulator study with $N=40$ participants, these systems were investigated with respect to their influence on measures of discomfort. Participants experienced different car following and braking scenarios on the highway with different time headways, with and without one of the passenger assistance systems. Based on the subjective measures, three systems were identified as particularly useful in reducing discomfort. For the best of these proposals, more than $63 \%$ of the passengers confirmed the usefulness of the approach and reported an interest in using it in their vehicle. This demonstrates significant opportunities to improve the everyday driving experience beyond classical assistant systems by explicitly taking into account the needs of the passengers.
\end{abstract}

Keywords: Discomfort, passenger, assistant systems, human machine interface, simulator study

\section{$1 \quad$ Introduction and Related Work}

Many people know the feeling of being a front seat passenger, experiencing situations in which they would have wished to have access to their own brake pedal, for example when a driver is distracted while following too close. Such events usually result in a feeling of discomfort. The Oxford English Dictionary (Stevenson \& Lindberg, 2017) defines "discomfort" as a feeling of "slight pain" or "to make (someone) feel uneasy, anxious or embarrassed". The definition of discomfort shows a connection to emotions like fear and to social emotions like embarrassment. Similar to these emotions, feelings of discomfort are a result of the evaluation of internal and external stimuli and signal motivational significance (Bower \& Cohen, 1982; Lazarus, 1982; Leventhal \& Scherer, 1987). In the case of the passenger, this means that an uncomfortable feeling shows that the experienced situation might be safety critical in the eyes of the passenger and disturbs their intention to experience a relaxed ride. Discomfort can arise on multiple levels - a physiological level such as caused by uncomfortable seats, a perceptual level including motion sickness, a psychological level that involves more complex environmental situations and a social level, reflecting interactions between humans. In this study, the focus will be on psychological discomfort. Previous interview and questionnaire studies (Ittner et al., 2020) showed that $88 \%$ of passengers experienced an uncomfortable situation at least once, frequently caused by fast driving and close following. Based on these results and well-established psychological models, like the feedback-loop model by Carver and Scheier (2002) and the transactional stress model by Lazarus and Folkman (1984), a cognitive model of causes for passenger discomfort was proposed (Ittner et al., 2020). This model tries to explain why passengers experience discomfort in situations that are evaluated as not critical by the driver, assuming that the driver would change the situation otherwise. This reasoning explicitly excludes objectively safety critical situations, as negative feelings would be fully appropriate in these cases. The model focuses on two main differences between driver and passenger leading to passenger 
discomfort. Firstly, a difference in the estimation of the situation between driver and passenger caused by limited or missing information. Secondly, the passenger's limited control over the vehicle.

So far, there are no assistance systems for passengers to support them in such situations. Generally, the passenger has played only a small or no role in the development of human machine interfaces (HMIs) for road vehicles and there are only a few HMIs specifically designed for them. Most of these systems focus on infotainment or on information for the passenger which help him to support the driver (Perterer et al., 2015; Trösterer et al., 2015; Maurer et al., 2014). In the studies by Maurer et al. (2014) and Trösterer et al. (2015) for example, they visualized the front seat passenger's gaze, augmented in the rendered view of a simulation, to improve communication and avoid misunderstandings with the driver during demanding situations like navigation or upcoming hazards. This takeover of tasks such as navigation by the passenger is intended to relieve the driver during rides. While a prototype of this HMI has been proposed by Maurer et al. (2014), Trösterer et al. (2015) compared this "HUD"-HMI to a more reduced version where an LED strip was attached along the bottom of the windshield. The passenger could influence with his/her gaze where LEDs light up to draw the driver's attention to that spot. They evaluated these two assistant systems regarding helpfulness for the driver, driving performance, or visualization accuracy (accuracy with which the passenger could highlight targets for the driver) with participant pairs of driver and a passenger. The results showed that the visualization accuracy was higher for the HUD system than for the LED system. Additionally, the passengers had the impression that the driver was significantly less satisfied with their navigation advice and that it was less helpful for the driver when they used the LED system. Perterer et al. (2015) investigated the influence of a more detailed navigation display for the passenger to take care of the navigation task. Aside from regular navigation information, the system showed additional information such as upcoming hazards. In a first explorative study, $75 \%$ of the drivers described the assistance by the passenger with the assistant system as relieving. However, none of these studies evaluated the effect on passenger discomfort and the proposed HMIs did not aim at providing information or other means to directly improve the driving experience of the passenger.

In contrast to this, human machine interfaces for driver assistance are well established and have been investigated in detail in many studies so far (Adell et al., 2008; Charissis \& Papanastasiou, 2010; Aydogdu et al., 2019; Winkler et al. 2018; Hofauer et al. 2018; Rittger et al. 2018). The goal of such HMIs is to support the driver by providing different types of information that help him in performing the driving task and avoid unwanted situations. This transparency of information can also have a positive impact on the comfort of drivers. In a Study by Charissis \& Papanastasiou (2010), they highlighted other vehicles on the road in a head-up-display (HUD), increasing spatial awareness and reducing response times under low visibility conditions. They compared this HUD with a conventional head-down-display (HDD) that highlighted nothing. Charissis \& Papanastasiou (2010) could show that participants had fewer collisions using the HUD, an improved response time, and $90 \%$ of the participants stated that they think that the system reduced their stress during the low visibility conditions. Driver HMIs are also used to visualize processes of active driver assistance systems which are not directly visible for the driver (e.g. activity and system status of an adaptive cruise control (ACC) or lane keeping assistant system (LKAS)). Aydogdu et al. (2019) compared an HUD with an HDD for an LKAS. Conventional HDDs show the system status of the LKAS for example with a green symbol when it is active and keeps the lane, when the symbol turns to grey the system is not active anymore. A pilot study (Aydogdu et al., 2019) showed that participants had troubles with keeping the lane and simultaneously monitor the system status with the HDD during sudden system drop-offs. The drop-offs were sometimes unexpected by the participants because they happened randomly and there was no warning when it happened. This lack of system status transparency made the participants feel stressed and not safe. In contrast, participants reported increased system transparency, increased safety, and decreased monitoring effort with the HUD. This shows that transparency not only has a generally positive effect on drivers but also on their comfort. 
This is also evident in the area of automated driving (AD) as almost all driving processes are not directly accessible by the driver anymore. Besides the purpose to show information about the system status of the vehicle, AD HMIs can also communicate more advanced information like planned maneuvers by the automated vehicle or system limits to prepare the driver to regain control. Since the driver in a fully automated vehicle has also no or limited control or influence on the vehicle behavior, similar to a passenger in conventional vehicles, results from this research area might be transferrable to passenger assistance HMIs. For AD HMIs, system transparency is achieved in different ways and its effect is mostly investigated for its positive effect on their trust in the automated vehicle. Chang et al. (2019) investigated the influence of different amounts of information on trust in an automated taxi. They visualized information about traffic controls, the intended path of the automated vehicle, or of other road users. Participants trusted the automated vehicle more when receiving this information. This relationship between system transparency and an increased user trust was also confirmed by other studies (e.g. Dadashi et al., 2013; Hoff \& Bashir, 2015; Yang et al., 2018). In AD, HMIs could also provide additional means to improve driving experience by offering specific ways to influence the driving process beyond providing transparency by showing information. A study by Frison et al. (2017) showed that providing a small level of voluntary control or influence options significantly improved the positive feelings of participants experiencing otherwise fully automated driving. These results suggest that with the help of HMIs, in addition to a transparent presentation of information, the provision of influence or control can have a positive impact on driving experience.

However, this positive effect of HMI information and control has not yet been studied for front-seat passengers. This paper will investigate if similar HMI concepts could be used to provide passengers with information or control that improve their driving experience. However, the main factor controlling the driving process in a passenger setting is the driver, which makes it much more difficult, compared to an AD setting, to provide relevant information in an HMI. This work aims to investigate potential sources of information and propose HMI candidates for passenger assistant systems with a focus on reducing the feeling of discomfort in specific situations. The passenger assistant system concepts are based on the cognitive passenger discomfort model proposed in Ittner et al. (2020), which will be described briefly in the next section. The existing concepts of driver assistance systems presented in the previous section will be discussed in the context of the model and adapted to passenger discomfort. After establishing a setup to reproduce passenger discomfort in a simulator, five concrete passenger assistance HMIs will be proposed and tested in a user study.

\section{Theory and Co-Driver Assistant System Concepts}

For the development of passenger assistant systems, it is relevant to know which mechanisms influence discomfort in order to provide appropriate information in relevant situations. According to the cognitive model introduced in Ittner et al. (2020, adapted in Figure 1), the driver regulates the driving task in a feedback-loop (Miller et al., 1960; Carver \& Scheier, 1998, 2002), where (s)he has full information about the own cognitive state at each point of the process (Figure 1 lower "Environment" box, without crosses). The driver has information about his or her attention focus (Input-Function), his or her own driving experience and driving style preferences (Reference Value), about their thresholds for estimation of the criticality of a situation (Comparator) and their planned actions (Output-Function). In contrast, the passenger has no or only limited information about this regulation process of the driver. This reduced or inaccessible information about the cognitive state of the driver is represented by the crosses in the "Environment" box in

Figure 1. This lack of information can lead to a different estimation of a situation's criticality between driver and passenger. If the result is an overestimation of criticality this might lead to passenger discomfort. According to the cognitive co-driver discomfort model, a lack of information can also negatively influence the trust in the driver in critical situations influencing their feeling of being exposed. This feeling of being exposed arises due to the passenger's limited ability to intervene in such situations and contributes to their discomfort (also represented by a cross in Figure 1). The published article (Ittner et al., 2020) also contains a more detailed description of the model. 


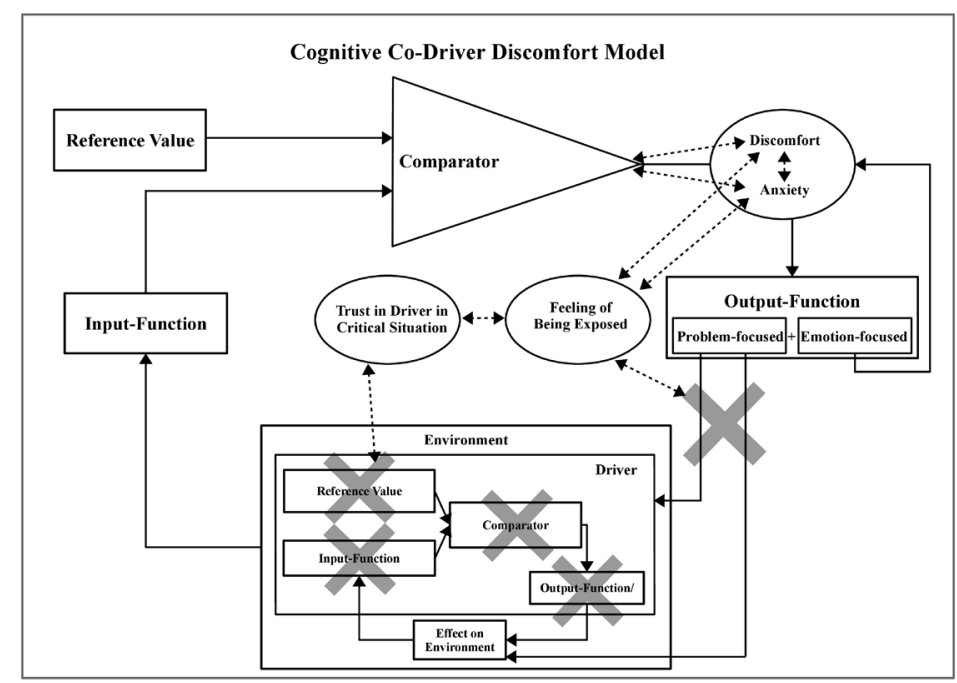

Figure 1: Cognitive co-driver discomfort model based on Ittner et al. (2020). Aspects in the model that are only sparsely or not available for passengers and therefore contribute to their discomfort are represented by gray crosses.

As mentioned in the introduction, this means that passenger discomfort can originate from (1) a lack of information about different aspects of the cognitive state of the driver, indicated by the gray crosses in the driver sub-model or (2) a lack of control/influence, respectively problem-focused coping possibilities, indicated by the gray cross after the output-function of the passenger. These basic mechanisms underlying the different assistance systems are applicable to different situations of the passenger. However, despite these same underlying mechanisms, it is more difficult to design a concept for an assistance system that is not only applicable in one specific situation but in different situations. It is also possible that the different mechanisms might have a different impact depending on the specific situation. Therefore, the derivation of the passenger assistant systems in this work is exemplarily explained and tested for the most frequently causing reasons close following and fast driving (Ittner et al., 2020). The following general hypothesis will be investigated in this work.

\section{H1: Displayed information or provided influence by the passenger assistant systems lead to a reduction of passenger discomfort compared to a baseline without assistance}

The relationship of assistance system information and control to a higher trust in the driver and a better situation assessment, as formulated in the model, are examined by correlations in order to validate the hypotheses.

\section{H1.1: Displayed information or provided influence will impact the passengers trust in the driver, their criticality estimation of the experienced situations, and their discomfort}

In order to investigate the influence of the individual mechanisms sub-hypotheses for each assistant system addressing these mechanisms are formulated in the next paragraphs. In the following, five concepts for passenger assistance systems will be presented. Each system aims to cover an aspect that contributes to passenger discomfort (symbolized by the gray crosses in the model).

Driver Input-Function: As the operator of the vehicle, the driver is part of the passenger's "environment" (Figure 1) and controls the vehicle based on the information he or she takes in (driver input function). In contrast to the driver, however, the passenger does not know if the driver is attentive and has seen all vehicles or traffic infrastructure that could become relevant for future driving. This uncertainty about the attentiveness of the driver can then lead to an overestimation of the situation's criticality and thus to discomfort. Due to the limited or lack of influence of the passenger, (s)he feels exposed to the situation, which can be amplified if there is little trust in the driver. In AD applications, commonly, the vehicle shares information about detected obstacles or hazards with the driver to improve take over times or take over 
performances (Yang et al., 2018). Naujoks et al. (2017) visualized detected front vehicles with an HMI designed similar to existing ACCs but there are also other means such as LED bars that light up to highlight detected hazards (Yang et al., 2018). For humans, visual perception is guided through eye movements, and therefore visualizing the targets of these eye movements can provide information about the perceived environment details, similar to what was done in the HMI studies by Trösterer et al. (2015) and Maurer et al. (2014). These ideas can be transferred to passenger HMI concepts. One possibility would be to highlight objects observed by the driver which could provide positive feedback about the attentiveness of the driver and reduce discomfort from uncertainties about detected obstacles. Alternatively, the system could analyze the gaze pattern of the driver with respect to objects deemed relevant and provide an abstracted signal of the results to the passenger. Using even higher means of abstraction, the system could measure the overall attention state of the driver, similar to conventional drowsiness detection systems (Ramzan et al., 2019), and communicate it with a passenger HMI. The first HMI concept will therefore target to investigate the following sub-hypothesis:

\section{H1.2: Passenger discomfort is reduced by information about the attentiveness of the driver compared to the baseline condition}

"Driver Attention Display" (At). This assistant system variation aims to communicate that the attention of the driver is focused on the relevant traffic situation (Figure 2). Regarding the use case investigated in this work, this information should provide the passenger positive feedback about the attentiveness of the driver during the distance regulation and his/her ability for a reaction to a sudden braking of the vehicle in front. Participants were told that the attentiveness of the driver was measured via electroencephalography (EEG) in the temporal area and via eye-tracking (Figure 2 (1)). It was explained that the data was used to determine how much the driver focused on the road. It was also explained that at different amounts of distraction the system state would change and that control glances in the mirrors were not treated as a distraction. We deliberately used the more visible measurement methods such as EEG or an eye-tracker, in which the camera is integrated into a pair of glasses. This should make it more convincing for the passenger that a functioning system fully maps the driver's attention. The attention focus was visualized on a display with a color-coded icon and a bar plot providing an intuition of the "amount of attention" (Figure 2 (3)(5)). The mapping of the different colors as explained to the participants was green for high attentiveness $(70 \%-100 \%)$, yellow icon (40\%) for a slight distraction of the driver, e. g. while changing the radio channel, and red for a critical and longer distraction of the driver $(<20 \%)$, as would be the case if the driver was reading a lengthy message on his cell phone. However, to ensure reproducibility, the driver's attentiveness was not computed online but defined based on the situation. In addition, as the main interest was to evaluate the system's potential to reduce discomfort, during the test scenarios the attention focus was constantly set to a high constant value, only providing positive information to the passengers. During the test scenarios, participants exclusively saw the green icons (Figure 2 (3) or (2)). The display showing this information was installed on the dashboard in front of the passenger (Figure 2 (2)).

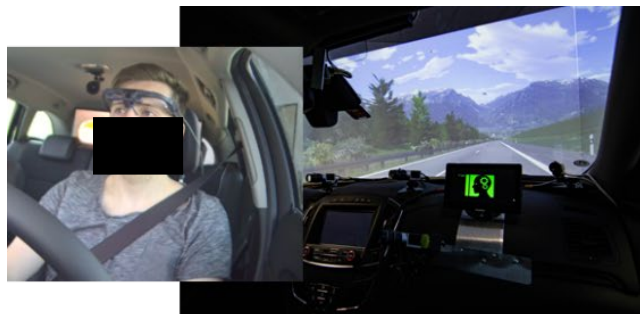

(1)
(2)

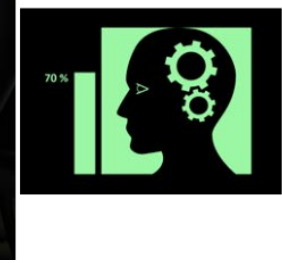

(3)

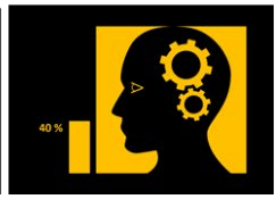

(4)

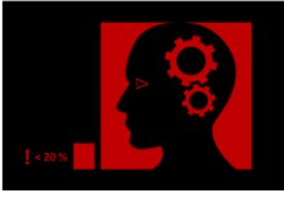

(5)

Figure 2: "Driver Attention Display" - (1) driver with eye-tracking glasses and two EEG electrodes in the temporal area. (2) Passenger display on the dashboard, showing the attention status of the driver. (3) - (5) Icon used for respectively high (70 \% $100 \%)$, medium (40\%) and low/critical attentiveness of the driver.

Reference value: According to the cognitive passenger discomfort model, passengers might have no or limited information about the driver's reference values, depending on familiarity with the driver. Reference 
values are used to ground the perceived input and are often formed through experience or habits (e.g. their preferred driving style, their driving experience, or accident history). In the context of the driving task, the reference value could be the distance to the car in front that the driver is used to, or a driving speed that (s)he perceives as comfortable or familiarity with certain aspects of an environment. There exist studies that showed that drivers who prefer smaller THWs to front vehicles are often more skilled in braking control (Winsum \& Heino, 1996). Transparency about these reference values could therefore be beneficial for the passenger's trust in a driver. In line with the passenger discomfort model, this transparency could also lead to a more accurate estimation of the situation criticality, which then could lead to lower passenger discomfort. Especially, if the passenger drives with an unknown driver like a taxi driver or with a lift, this type of information could be useful to better estimate situations. A study by Khastgir et al. (2018) showed that information about an automated vehicle's system capabilities has led to higher trust in the system, regardless of whether the capabilities or limitations were high or low. A possibility for passenger assistance would be to provide information or statistics about these driver's reference values or skills. Possible HMI concepts could provide static information for example about the experience of a driver or more detailed information matching the current driving situation such as the average THW from the driver's past highway drives. For this assistant system the following sub-hypothesis will be evaluated:

\section{H1.3: Passenger discomfort is reduced by information about reference values based the driver's experience compared to the baseline condition}

"Preferred THW HUD" (PTHW). Based on the above-mentioned findings from the passenger model and related work, this assistant system tries to communicate a reference value that a driver uses to evaluate following distances. This was approached by providing information through a simulated head-up display (HUD) visible to both driver and passenger. It was rendered as a semi-transparent bar on the road between the ego-vehicle and the preceding car directly into the simulation environment. This type of visualization makes the information equally and intuitively available to both occupants. The semi-transparent bar had a blue color and a constant length (Figure 3). Participants were told that the bar's length corresponds to the distance the driver is usually using. During the instruction ride, the driver was asked to drive his/her personal preferred driving style and with a distance at which they know, they can still react in time to calibrate the system. However, as the driver was part of the experimental design, the actual preferred THW used during the calibration was the same (THW $=0.4 \mathrm{~s}$ ) for all participants and in every test scenario. Additionally, the passengers got the information that the driver is experienced and never had an accident. This aimed at creating the impression that the driver is able to react adequately even at small distances.

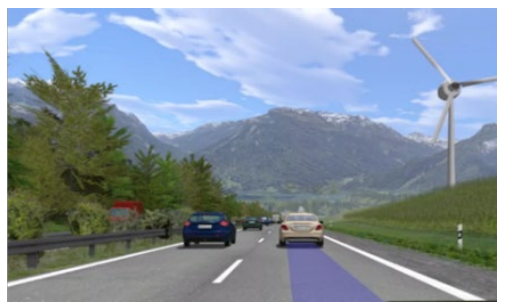

Figure 3: "Preferred THW HUD" - Visualization showing a driver experience THW of $0.4 \mathrm{~s}$.

Comparator: The cognitive passenger model implies another possibility that can influence the estimation of a situation's criticality, which can increase the passenger's discomfort. The passenger does not know how the driver assesses different situations. For example, if (s)he incorrectly considers the distance to a front vehicle to be sufficient or if it is rather the passenger that incorrectly estimates it as too small. In terms of the model, this means that the passenger does not know how the driver calculates the input with its reference value in the comparator. A possible solution would be an objective technical system that takes over the position of the driver's comparator displaying to the driver and passenger, for example, the legal minimum distance. If this information would be available to both driver and passenger, the latter could rely on the fact that they are both using the same comparator for their evaluations. Similarly, this was realized 
in a study by Israel et al. (2010). They visualized the HMI of an ACC in a HUD to give the driver feedback about actions of the ACC. The HMI showed information about the actual distance to a vehicle in front. This was realized with a partly green and red bar behind the front vehicle. When the red bar near the back of the front vehicle was reached the ACC started to brake. This type of information made it possible for the driver to better anticipate when the ACC will start to brake (i.e. know the ACC's reference value) and when (s)he should intervene if not. For the passenger, this type of information could have the same effect. This leads to the following sub-hypothesis investigated for this assistant system:

\section{H1.4: Passenger discomfort is reduced by explicit information about the safety thresholds provided to both the passenger and the driver compared to the baseline condition}

"Shared Safety Distance HUD" (SD). This passenger assistant system has the target to communicate to the passenger that (s)he has a shared understanding of a 'safe' distance to the front vehicle with the driver. The visualization was similar to the "Preferred THW HUD". The system also was implemented as a semitransparent bar which had a green color until a defined safety distance was undercut (Figure 4 left). When the safety distance was too small the bar changed its color to red (Figure 4 right). The participants received the explanation that the red color signals that the driver would no longer be able to react in time to a sudden break of the front vehicle given the current velocity and distance, and an accident could not be avoided. However, the participants always received positive feedback about the safety distance (green bar) during the test scenarios. This means that in the study the presented safety distance was adapted to match target setups for different experiments.
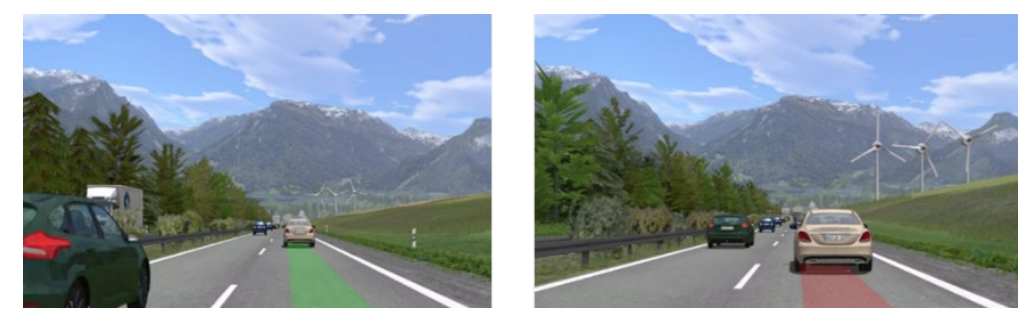

Figure 4: "Shared Safety Distance HUD" - Left: HUD when the safety distance is sufficient. Right: HUD when the distance is below safety threshold.

Driver Output-Function: The cognitive passenger model names another factor leading to passenger discomfort. Uncertainty regarding already executed actions or intentions of the driver. This can be the case in potentially critical situations when the driver is ready to brake while this cannot be incorporated into the evaluation of a passenger. A similar effect is caused by the time delay between an applied braking force and the perception of deceleration of a passenger. With an assistant system displaying actions or intentions of the driver, it would be easier for passengers to predict or more accurately evaluate the safety of the behavior of a driver. This transparency could lead to a more accurate estimation of the situation and to an increased trust in the driver. In a study by Löcken et al. (2016), information about the intentions of an automated vehicle improved the accuracy of the estimates of intentions and future maneuvers of the vehicle. Chang et al. (2019) also visualized information about the intended path of the automated vehicle or of other road users. Participants trusted the automated vehicle more when having access to such an HMI. Unfortunately, the early detection and visualization of human intentions is more difficult as compared to the plans of an automation system. One approach to reduce the perception delays could be to visualize the driver's foot position with respect to the brake pedal for the passenger. With this information, they could see that the driver is already prepared to brake or did already start braking. Thus, the hypothesis under investigation for this system is:

\section{H1.5: Passenger discomfort is reduced by information about the braking intentions of the driver compared to the baseline condition}


"Braking Information Display" (BI). The information from the previous paragraph leads to the suggestion that this assistant system should display information about driver actions relevant for the regulation of longitudinal velocity. The same display used for the "At" system was used to show the approximate position of the driver's foot with respect to brake and gas pedal to the passenger (Figure 5). The HMI used two Icons for positions on the gas pedal (grey): A foot on the gas pedal and a foot lifted from the gas pedal. For the brake pedal, three more icons were showing different braking intensities. Additionally, a vehicle icon was shown with three different brightness levels of the brake lights corresponding to the braking intensities. During manual driving, the display of the pictures (1) and (2) was controlled by the gas pedal pressure and the display of the other three pictures was controlled by the brake pedal pressure. When the driver used the ACC, the ACC signal was used to regulate which picture was displayed.

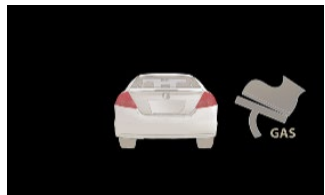

(1)

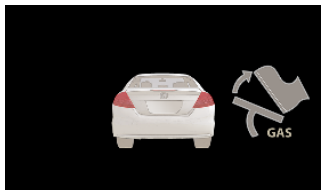

(2)

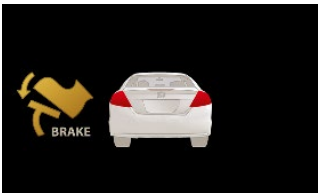

(3)

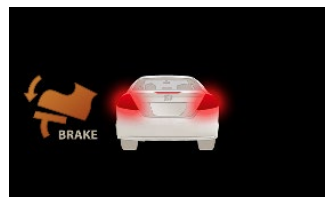

(4)

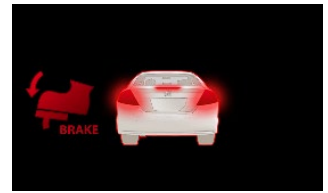

(5)

Figure 5: "Braking Information Display"- Icons shown when the driver has his foot on the gas pedal (1), releases the gas pedal (2), and applies different intensities of pressure to the brake pedal (3) - (5).

Limited Passenger Output-Function: When a passenger assesses a situation as more critical than the driver due to limited information (e.g. about the driver's input-/output-function, comparator or reference value), his/her ability to actively intervene or cope with situations in a problem-focused way is limited as they have no access to the vehicle controls. There is only an indirect possibility to influence the situation by asking the driver to change the driving behavior. However, the passenger has to rely on the driver following such a request. If this is not the case, the situation does not change and the discomfort stays high according to the passenger model. It might even add social discomfort. For that reason, some passengers might refrain from this coping option as the driver could understand this as a criticism of their driving style. Another way to cope with such situations is the passive emotion-focused way. This means that the passengers could try to change their emotions for example to reduce discomfort through distraction. Interviews by Ittner et al., (2020) showed that a large proportion of passengers used emotion-focused strategies (42\%) like breathing, grabbing a door handle, or distracting themselves. In comparison, the more problem-focused strategy of saying something to the driver or criticizing him/her (21\%) was used less frequently. Both strategy types showed mixed helpfulness. The limited possibility to cope with such situations can lead to a feeling of being exposed and supports passenger discomfort (Ittner et al., 2020). Providing additional means of control to the passengers could therefore reduce the feeling of being exposed and consequently discomfort. Results of a study by Frison et al. (2017) support this conclusion. They showed that AD without any control satisfied driver needs significantly less than manual driving and that the cooperative automation reduced the negative effects of pure automation. However, allowing a direct impact on driving maneuvers, like it was used in that study, is much easier to provide between a driver and an automated vehicle, while it might strongly interfere with the driving task in the passenger context. There are studies (Hesse et al., 2013; Schieben et al., 2014) that show that automatically initiated steering interventions in conventional vehicles can avoid collisions, but drivers tend to counter steer or hold the steering wheel during these interferences. Some participants in the study by Schieben et al. (2014) explained that they found the automatic intervention frightening or feared a collision with the traffic on the opposite lane. For passenger assistance, it might be a better alternative to provide additional means of indirect control. The passenger could, for example, have an own brake pedal, which does not control the vehicle but provides the driver with a signal indicating a braking suggestion. However, such communication with the driver might be more successful if it would be based on a situational context instead of a direct action recommendation. The passenger could trigger the highlighting of relevant traffic participants to the driver as in Trösterer et al. (2015) or provide information about safety distances. 
In the context of the use case formulated at the beginning of this section and the cognitive passenger model the sub-hypothesis is as follows:

\section{H1.6: Passenger discomfort is reduced by having influence on the safety distance compared to the baseline condition}

“Active Distance Influence HUD” (Button/Bu). The visualization of this system is similar to the shared safety distance assistant system. The functional difference is that the passenger could decide when the color of the bar would change from gray to red (and back) using a hidden button (Figure 6). With this, they can signal the driver whenever they think that the safety distance feels too small. By pressing the button again, the passenger could change the color from red to gray again to show the driver that the distance is sufficient. The participants got the instruction that the driver was not informed that they have such a controller, but that the driver got the same instruction as for the shared safety distance system. This should communicate, that the driver would have the impression that a displayed safety distance is based on an objective measure.

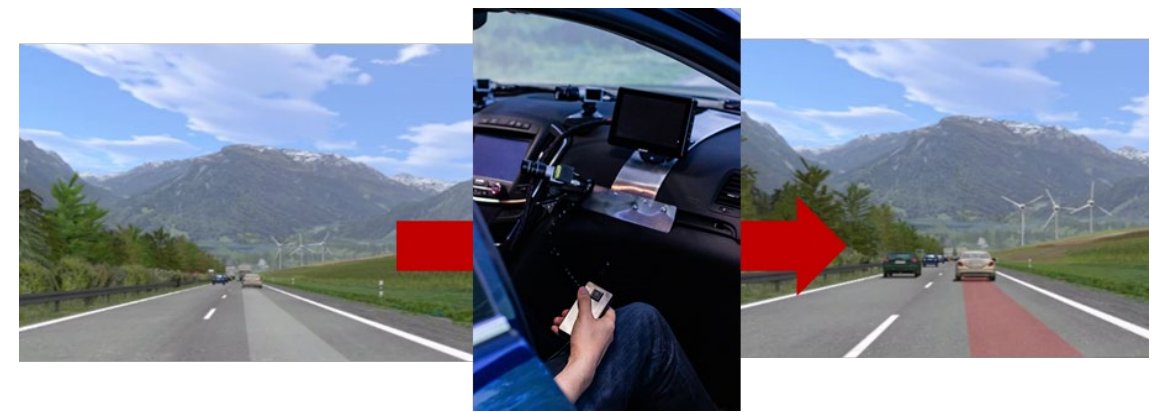

Figure 6: “Active Distance Influence HUD”- Left: HUD when the passenger does not intervene. Middle: Passenger intervention using a handheld controller. Right: HUD after the passenger pressed the button, independent of actual distance.

\section{$3 \quad$ Feasibility Study - Passenger Discomfort}

To examine the impact of the proposed passenger assistant systems on passenger discomfort a simulator study was set up. For this research question, a simulator study has the advantage that influences of the environment, like weather or traffic conditions, can be controlled and therefore, scenarios can be replicated exactly for each participant. However, the results of such a study can only be valid, if it is possible to induce passenger discomfort in a simulator. Since prior research only used surveys or real driving studies with passengers, but so far, no simulator studies investigating passenger discomfort, we decided to investigate if passengers can experience discomfort in a simulator and if they can experience it without becoming motion sick in a feasibility study.

\subsection{Methods}

$N=9$ participants ( $n=5$ male; $n=4$ female) took part in the feasibility study. They were frequent passengers who were recruited from prior participants in the interview study reported in (Ittner et al 2020). $22 \%$ were passengers 3-5 times per week, $56 \%$ 1-3 times per month, and $22 \%$ less than once per month.

The static simulator that was used consists of a full-body production car in a closed room facing five screens surrounding the vehicle (Figure 7). The simulation is done with the software SILAB $^{1}$ (Impressions can be seen in Figures 2-6). The frontal field of view covers an angle of $300^{\circ}$ (horizontal) and $47^{\circ}$ (vertical). The rendered scene is projected onto the screens using five projectors with a resolution of $1400 \mathrm{x} 1050$ pixels each. The side mirrors are replaced by LCD-displays showing the simulated rearview. The rearview mirror is a conventional rearview mirror and shows the virtual environment via another LCD-display in the trunk. Since the simulator was originally designed for drivers the projected perspective is oriented towards the

\footnotetext{
${ }^{1}$ https://wivw.de/en/silab
} 
driver seat. This could lead to higher chances of experiencing simulator sickness on the passenger seat, due to the different angle of view for motions in the simulation which differ from motions as they are usually perceived and expected. To avoid simulator sickness caused by these different viewing angles, the projection of the simulation was adjusted towards the passenger's position.

The driver was an instructed expert of the WIVW GmbH, who was tested to not be susceptible to simulator sickness in this passengerfocused setting. The driver was treated like a participant in front of the passenger participants (e.g. it was explained that the driver will be interviewed by another experimenter in the preparation room after the experiment). The driving behavior in all scenarios was prerecorded so that it was exactly the same for every participant. During a run, the driver pretended to steer the vehicle himself/herself. This should prevent that the participants feel less discomfort as they could get the impression that the driver knows what will happen next.

In the design of the uncomfortable situations, results from previous interviews (Ittner et al., 2020) were considered. In these interviews,

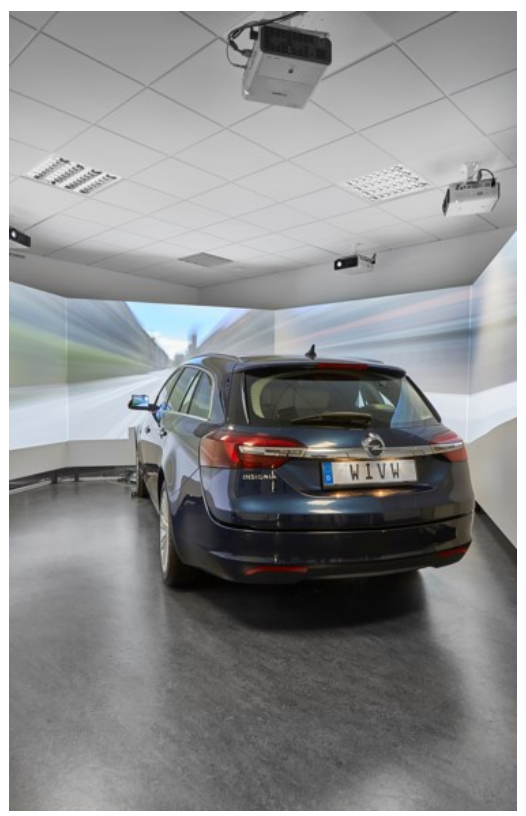

Figure 7: Static simulator with a full body production car. most participants named close following and high velocities as prominent reasons for their discomfort. Therefore, Following and Braking scenarios were designed. Both types of scenarios were experienced on a 2-lane autobahn, to investigate the effect of higher velocities on passenger discomfort, and with a constant headway. In the Following scenario, the driver was driving behind several cars on the left lane with 120 $\mathrm{km} / \mathrm{h}$ while the right lane was crowded with vehicles and trucks so that the driver was forced to stay on the left lane. The braking scenario was the same as the Following scenario except that a Truck changed its lane to the left and forced the cars in front of the ego-vehicle to brake strongly. Therefore, the ego-vehicle was likewise forced to brake. These two scenario types were experienced by the participants with a short, safety critical time-head way (THW: Distance/velocity of follower; THW $=0.5 \mathrm{~s}$ ) and with a larger, safe distance $(\mathrm{THW}=1.5 \mathrm{~s})$. In the Braking scenario, the front vehicle decelerated with max. $-4.5 \mathrm{~m} / \mathrm{s}^{2}$ from approx. 120 $\mathrm{km} / \mathrm{h}$ to $90 \mathrm{~km} / \mathrm{h}$. We selected relatively short THWs, as it is known, that situations appear less critical in a static simulator than in reality due to a lack of motion cues, such as vestibular information, which are important for speed control or steering (Reymond et al., 2001; Wierwille et al., 1983). The $2 * 2=4$ scenarios were presented to the participants in a randomized order (approx. 20 minutes for each part).

After each situation, the experimenter interviewed the participants inside the car. To measure the participants' subjective discomfort, they were asked to rate it on a 16-point $(0=$ not at all $\ldots 15=$ very strong $)$ category subdivision scale after each situation. Simulator Sickness was measured with the Simulator Sickness Questionnaire on a 4-point scale (none, slight, moderate, and severe intensity of simulator sickness symptoms) (SSQ; Kennedy, Berbaum, \& Smith, 1993; Kennedy, Lane, Berbaum, \& Lilienthal, 1993). To control for possible symptoms which are not induced by the simulator, participants completed the SSQ before the procedure and after the whole procedure. At the end of the study, the participant was informed about the fact that the driver was following an instructed driving style.

\subsection{Results}

Figure 8 shows that most participants felt strongly uncomfortable during short THWs in the Braking scenario on the autobahn ( $m=11.56, s d=3.21)$ as compared to the longer THWs $(m=5.11, s d=3.41)$. Detailed tests showed that this difference is significant (Asymptotic Wilcoxon-Test: $z=-2.68, p<.05, n=9$, $\eta^{2}=.80$ ). The same relation was found for the Following scenarios (Asymptotic Wilcoxon-Test: $z=-2.53$, $\left.p<.05, n=9, \eta^{2}=.71\right)$. In the Following scenario participants experienced medium discomfort during short THWs $(m=9.00, s d=3.20)$ and low discomfort during long THWs $(m=5.89, s d=3.37)$. 


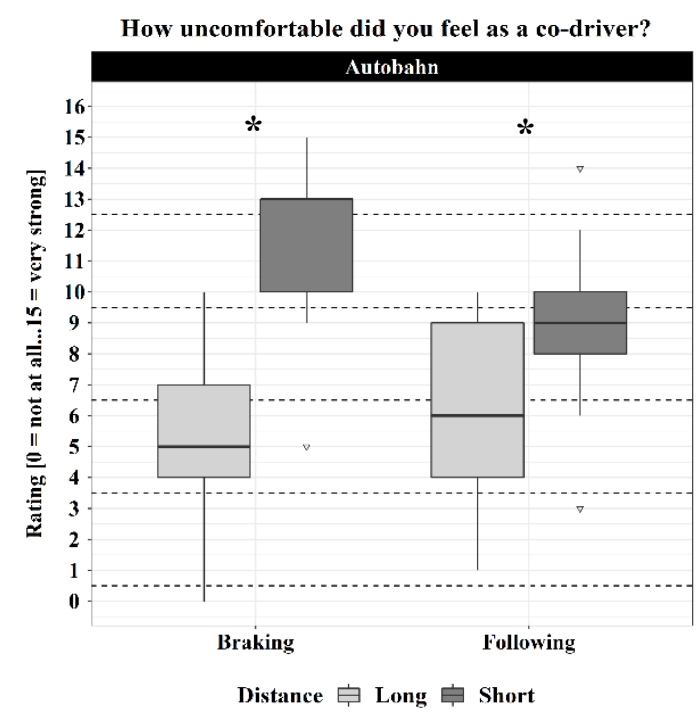

Figure 8: Discomfort ratings for each situation and distance for the autobahn environment. Significant effects are marked with $*$ Box range $=Q 1$ to $Q 3$. Whiskers $=1.5 * I Q R$.

All participants completed their runs without interruption of the procedure due to simulator sickness. The mean Total SSQ Scores before $(m=20.59, s d=24.39)$ and after $(m=60.78, s d=87.83)$ the procedure showed a slight increase of the symptom severity, but the difference was not significant (Asymptotic Wilcoxon-Test: $z=-1.52$, n. s., $n=10$ ). Only a few participants showed slight symptoms after the procedure $N=1$ participant reported severe salivation and another $N=1$ reported moderate eyestrain after the last session. The more simulator sickness specific symptom Nausea was also only reported to a small degree by $N=1$ participant. The driver showed no signs of simulator sickness during the whole study.

\subsection{Discussion}

The results show that participants can experience discomfort in a static simulator without experiencing simulator sickness on the front passenger seat when the field of view is adapted accordingly. The most effective settings were autobahn scenarios with close following of other vehicles, especially when the driver was suddenly forced to brake under these conditions. Although the discomfort ratings of the long THW scenarios were significantly lower than the ratings of the short THW scenarios, the mean values show that, with the exception of a few participants, the passengers also experienced some discomfort in these situations. The same scenarios were also tested in a city environment (not reported here) which showed similar results regarding passenger discomfort. However, in the following, we will continue to use the autobahn setting.

\section{User Study - Passenger Assistance}

The user study then investigated the influence of the various assistance systems on the passenger and compared the different assistance systems in this respect.

\subsection{Methods}

\subsubsection{Sample}

The study in the static simulator was conducted with $N=40$ participants ( $n=21$ female and $n=19$ male) with an age between 21 and 68 years ( $m=43.2$ years, $s d=14.2$ years). $65 \%$ of the participants were daily drivers and $50 \%$ of the sample were passengers at least once per week.

\subsubsection{Scenarios}

The scenarios used in this simulator study were similar to the autobahn scenarios in the feasibility study. On the autobahn, the driver followed a car on the left or right lane with $120 \mathrm{~km} / \mathrm{h}$ while the other lane was crowded with vehicles and trucks. At one point a truck or vehicle changed its lane to the left or right (depending on which lane the ego-vehicle was) and forced the car in front of the ego-vehicle to brake 
strongly. Therefore, the driver of the ego-vehicle was also forced to brake. Based on the results of the feasibility study and on the experience with static simulators, we wanted to focus on situations with a higher probability of causing discomfort in the participants. Therefore, after the feasibility study, we decided to focus on shorter THWs and increased the braking deceleration of the front vehicle. In the Braking scenario, the front vehicle decelerated with max. $-12.5 \mathrm{~m} / \mathrm{s}^{2}$ from approx. $120 \mathrm{~km} / \mathrm{h}$ to approx. $70 \mathrm{~km} / \mathrm{h}$ on the autobahn The Following scenario was the same as the Braking scenario except that no truck or vehicle changed lanes. Each of the two trips had six permutated scenarios consisting of three Braking scenarios and three Following scenarios with the time headways THW $=[0.3 \mathrm{~s}, 0.6 \mathrm{~s}, 0.9 \mathrm{~s}]$ (Table 1). At the beginning of each Following or Braking scenario, there was a part in which the driver approached some vehicles on his/her lane. After driving through a scenario, a short section without traffic followed in order to connect the scenarios. All hypotheses were investigated for both the Braking and Following scenarios.

Table 1

Structure of the two trips

\begin{tabular}{|c|c|c|c|c|}
\hline \multirow{3}{*}{ N Scenarios } & \multicolumn{2}{|c|}{ 1. Trip } & \multicolumn{2}{|c|}{ 2. Trip } \\
\hline & \multicolumn{2}{|c|}{6} & \multicolumn{2}{|c|}{6} \\
\hline & 3 & 3 & 3 & 3 \\
\hline Scenario & Following & Braking & Following & Braking \\
\hline THW & $0.3 \mathrm{~s}, 0.6 \mathrm{~s}, 0.9 \mathrm{~s}$ & $0.3 \mathrm{~s}, 0.6 \mathrm{~s}, 0.9 \mathrm{~s}$ & $0.3 \mathrm{~s}, 0.6 \mathrm{~s}, 0.9 \mathrm{~s}$ & $0.3 \mathrm{~s}, 0.6 \mathrm{~s}, 0.9 \mathrm{~s}$ \\
\hline
\end{tabular}

\subsubsection{Procedure}

The study was conducted in the same simulator as the feasibility study. To show the feedback of the assistant system "attention" and "braking info" an additional display was installed in front of the passenger at the height of the airbag. For the Active Distance Influence assistant system, a button was attached to the right side of the front passenger seat. The duration of one run was $1.5 \mathrm{~h}$ per participant, in which they experienced two trips of 20 minutes as a passenger on a two-lane Autobahn. The remaining time of 50 minutes was used for the introduction of the assistant systems and interviews after the session. Every participant experienced one trip without assistance and one with one of the passenger assistant systems (sample of $N=8$ participants per assistant system). For an overview of the study design, see Table 2. The ordering of the two trips was randomized. Before the trip with assistance, driver and passenger experienced all HMI states during a short instruction ride. During the road sections which connected the scenarios, the passengers answered the scenario-specific questions. The questions were organized in a folder that allowed them to answer the questions covertly in front of the driver. This should prevent the participants from giving lenient ratings in the situations because the driver could see a negative evaluation and take it as criticism.

Table 2

Study design with an overview of the distribution of the subjects

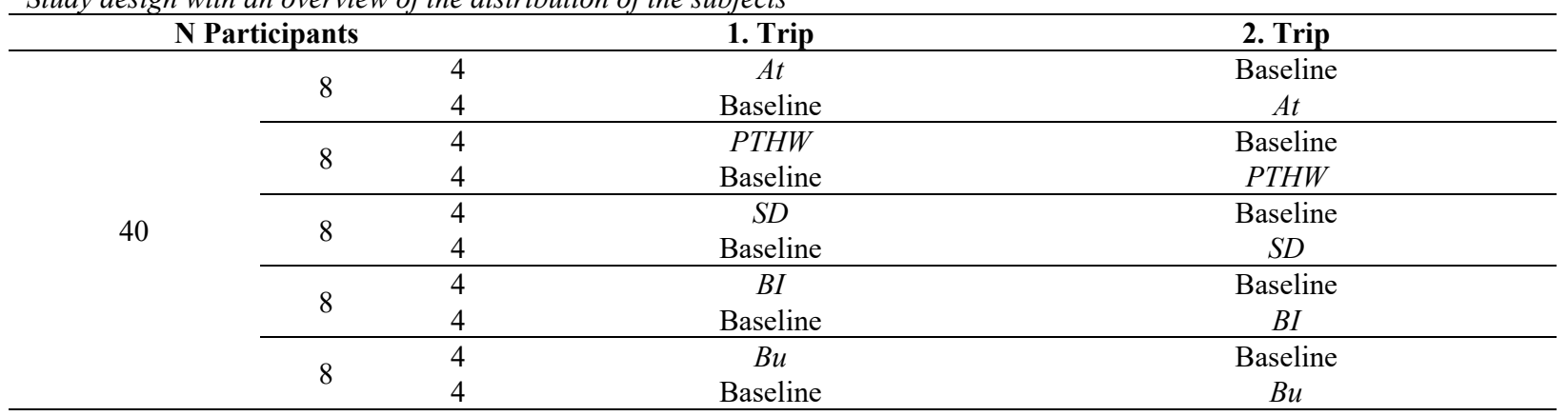

Note. Baseline $=$ no assistance.

Like it was done in the feasibility study, the drivers were instructed co-workers, but this was not revealed to the participants at the start of the study. The drivers drove with an instructed driving style and a simulated adaptive cruise control (ACC) function activated. In presence of the participants, the drivers were instructed that it is their own decision whether to follow the system's feedback or not. However, for the drivers, it was 
also part of the instructed driving style to follow the feedback of the assistant systems at all times. This method was used to guarantee the greatest possible standardization and comparability of the driving style for each participant while allowing for uncertainty about the driver's reactions. At the end of the study, participants were informed about the fact that the driver followed an instructed driving style.

\subsubsection{Dependent Variables}

As in the feasibility study, after each scenario, subjective discomfort was rated on a 16-point category subdivision scale $(0=$ not at all...15 = very strong). In the same way, participants were asked to estimate how safety critical the situation was, their trust in the driver and how much they felt exposed to the situation (see Table A.3 in the appendix for the complete formulations of the items). During rides with an assistant system, the participants also rated after each scenario how helpful the presented information/the influence means was in general and how helpful the assistant system was to better assess the situation (Figure 9). In the post inquiry, participants rated how helpful the assistant system was to reduce their discomfort in general with the same 16-point category subdivision scale and they were asked if they would use the experienced co-driver assistant system in a future car.

\begin{tabular}{|c|c|c|c|c|c|c|c|c|c|c|c|c|c|c|c|}
\hline $\begin{array}{l}\text { not helpful at } \\
\text { all }\end{array}$ & \multicolumn{3}{|c|}{ marginally helpful } & \multicolumn{3}{|c|}{ slightly helpful } & \multicolumn{3}{|c|}{ medium helpful } & \multicolumn{3}{|c|}{ very helpful } & \multicolumn{3}{|c|}{ extremely helpful } \\
\hline 0 & 1 & 2 & 3 & 4 & 5 & 6 & 7 & 8 & 9 & 10 & 11 & 12 & 13 & 14 & 15 \\
\hline
\end{tabular}

Figure 9:16-point category subdivision scale used for the rating of system helpfulness after each scenario and in the post inquiry.

\subsection{Results}

\subsubsection{Online Ratings}

Figure 10 (left) shows the distributions of the information/influence helpfulness ratings overall 48 scenarios driven by $N=8$ participants ( 6 scenarios per participant) for each assistant system. The participants rated information presented by the "Attention" assistant system as very or extremely helpful in $69 \%$ of the scenarios and in an additional $15 \%$ of the scenarios the presented information of the system was rated as medium helpful. Similarly, in $63 \%$ of the scenarios, the participants rated the information and influence possibility provided by the "Button" system as very or extremely helpful, and in $11 \%$ of the scenarios as medium helpful. For the "Safety Distance" assistant system, the proportion of scenarios in which the information was rated as very or extremely helpful was slightly lower with $52 \%$. On the other hand, the proportion of scenarios in which the information was rated as moderately helpful was higher with $25 \%$. The information presented by the other two assistant systems was very or extremely in less than $50 \%$ (44\% for "Braking Information" and $39 \%$ "Preferred THW") of the experienced scenarios. In $17 \%$ of the scenarios, the braking information was considered medium helpful and in $25 \%$ of the scenarios, this was true for information about the preferred THW.

The distribution of ratings for the question how helpful the assistance systems were to better assess the situation were similar (Figure 10 right). The assistant system "At" was again very or extremely helpful in most of the scenarios (73\%) followed by the assistant systems "SD" $(56 \%)$ and "Bu" (46\%). The other two assistant systems were very or extremely helpful in fewer scenarios ( $44 \%$ of the situations for "BI" and "PTHW" each). 


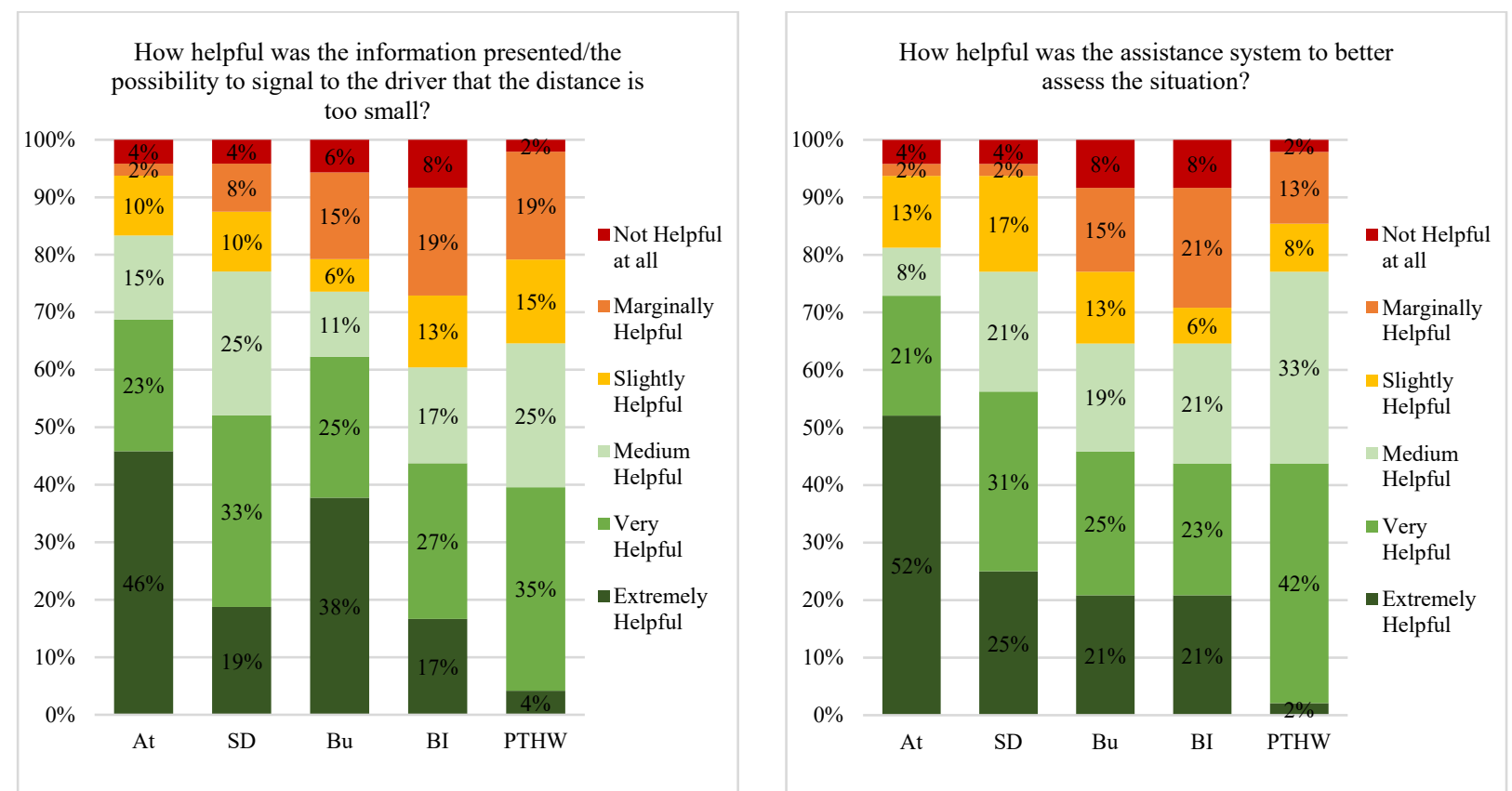

Figure 10: Distribution of scenario specific ratings regarding (left) the helpfulness of the present information respectively the possibility to have influence, and (right) the helpfulness of the assistance system to better assess the situations (right).

Correlations were used to investigate the influence of the presented information or provided control by the assistant systems on the participants' discomfort or trust in the situations. Table 3 shows for each assistant system the correlations between the helpfulness of the information/the possibility to have influence and the perceived criticality of the scenario, the experienced discomfort, the trust in the driver, and the helpfulness of the assistant system to better assess the situation. There are significant relations between the helpfulness of the information displayed by the "attention" assistant system and all other variables. The more helpful the information was rated, the less critical the scenarios were estimated and the less uncomfortable the participants felt. They also trusted the driver more and rated the assistant system as more helpful to better assess the situation. The relation between the helpfulness of the information provided by the assistant systems and the helpfulness of the systems to better assess the situation was found for all variations. There were also significant connections for the "Bu" system to the variables "Criticality", "Discomfort", and "Trust in the driver". However, the direction of this relation was contrary to the connection found for the attention assistant system. The more helpful the participant rated the possibility to signal to the driver that the TWH is too small, the more critical and uncomfortable the situations were assessed. Additionally, the more the trust in the driver was reduced, the more helpful the possibility to intervene in the scenarios was rated. The other assistant system variations showed no relation to the other variables.
Table 3

Pearson correlations between the helpfulness of the displayed information/provided influence and the variables criticality, discomfort, trust in the driver and the helpfulness of the assistant system to better estimate the situation by assistant system.

\begin{tabular}{llcc}
\hline $\begin{array}{l}\text { System } \\
(N=\mathbf{4 8})\end{array}$ & Variables & $\begin{array}{c}\text { Info/Influence } \\
\text { Helpfulness } \\
\text { (Pearson) }\end{array}$ & $\mathbf{p}$ \\
\hline At & Criticality & $\mathrm{r}=-.63$ & $<.01$ \\
& Discomfort & $\mathrm{r}=-.65$ & $<.01$ \\
& Trust & $\mathrm{r}=.70$ & $<.01$ \\
& Estimate & $\mathrm{r}=.97$ & $<.01$ \\
\hline \multirow{3}{*}{ SD } & Criticality & $\mathrm{r}=.17$ & $\mathrm{n} . \mathrm{s}$. \\
& Discomfort & $\mathrm{r}=.10$ & $\mathrm{n} . \mathrm{s}$. \\
& Trust & $\mathrm{r}=-.13$ & $\mathrm{n} . \mathrm{s}$. \\
Bu & Estimate & $\mathrm{r}=.96$ & $<.01$ \\
\hline & Criticality & $\mathrm{r}=.41$ & $<.01$ \\
& Discomfort & $\mathrm{r}=.50$ & $<.01$ \\
& Trust & $\mathrm{r}=-.43$ & $<.01$ \\
BI & Estimate & $\mathrm{r}=.89$ & $<.01$ \\
\hline & Criticality & $\mathrm{r}=.13$ & $\mathrm{n} . \mathrm{s}$. \\
& Discomfort & $\mathrm{r}=.04$ & $\mathrm{n} . \mathrm{s}$. \\
& Trust & $\mathrm{r}=.12$ & $\mathrm{n} . \mathrm{s}$. \\
& Estimate & $\mathrm{r}=.98$ & $<.01$ \\
\hline & Criticality & $\mathrm{r}=-.11$ & $\mathrm{n} . \mathrm{s}$. \\
& Discomfort & $\mathrm{r}=-.08$ & $\mathrm{n} . \mathrm{s}$. \\
& Trust & $\mathrm{r}=.16$ & $\mathrm{n} . \mathrm{s}$. \\
& Estimate & $\mathrm{r}=.87$ & $<.01$ \\
\hline
\end{tabular}

Note. n.s. $=$ not significant $\mathrm{p}>=.05$ 
The $N=40$ participants experienced $N=6$ scenarios per person resulting in $N=240$ scenarios with assistant system and the same number without assistant system. In a first step, the main effect of reducing discomfort was investigated for all assistant systems together. In total, participants experienced a reduction of discomfort in comparison to the same scenarios without assistant system in $N=118(49 \%)$ cases. In $N=$ $76(32 \%)$ scenarios there was no change of the discomfort rating and in $N=45$ scenarios $(19 \%)$ there was an increase of discomfort. Asymptotic Wilcoxon-Tests showed a significant reduction of discomfort by the assistant systems $\left(z=-4.75, p<.001, n=239, \eta^{2}=.094\right)$. This was found for assistant systems providing only information (Asymptotic Wilcoxon-Test: $z=-3.54, p<.001, n=191, \eta^{2}=.066$ ) and for the assistant system which provided control (Asymptotic Wilcoxon-Test: $z=-3.28, p<.01, n=48, \eta^{2}=.22$ ).

In the next step, detailed tests were used to investigate the effects of each assistant system in the Braking and Following scenarios during different THWs. Figure 11 shows for each system the discomfort ratings by the participants during the Braking scenarios. For short THWs, a significant reduction of discomfort was only found for the "Bu" system, while for medium and long THWs there was a significant reduction by the "At" and the "SD" systems (Table 4). The "BI" assistant system seemed to even increase discomfort at short THWs. The system "Preferred THW" showed no significant reduction of the passenger's discomfort for any setting.

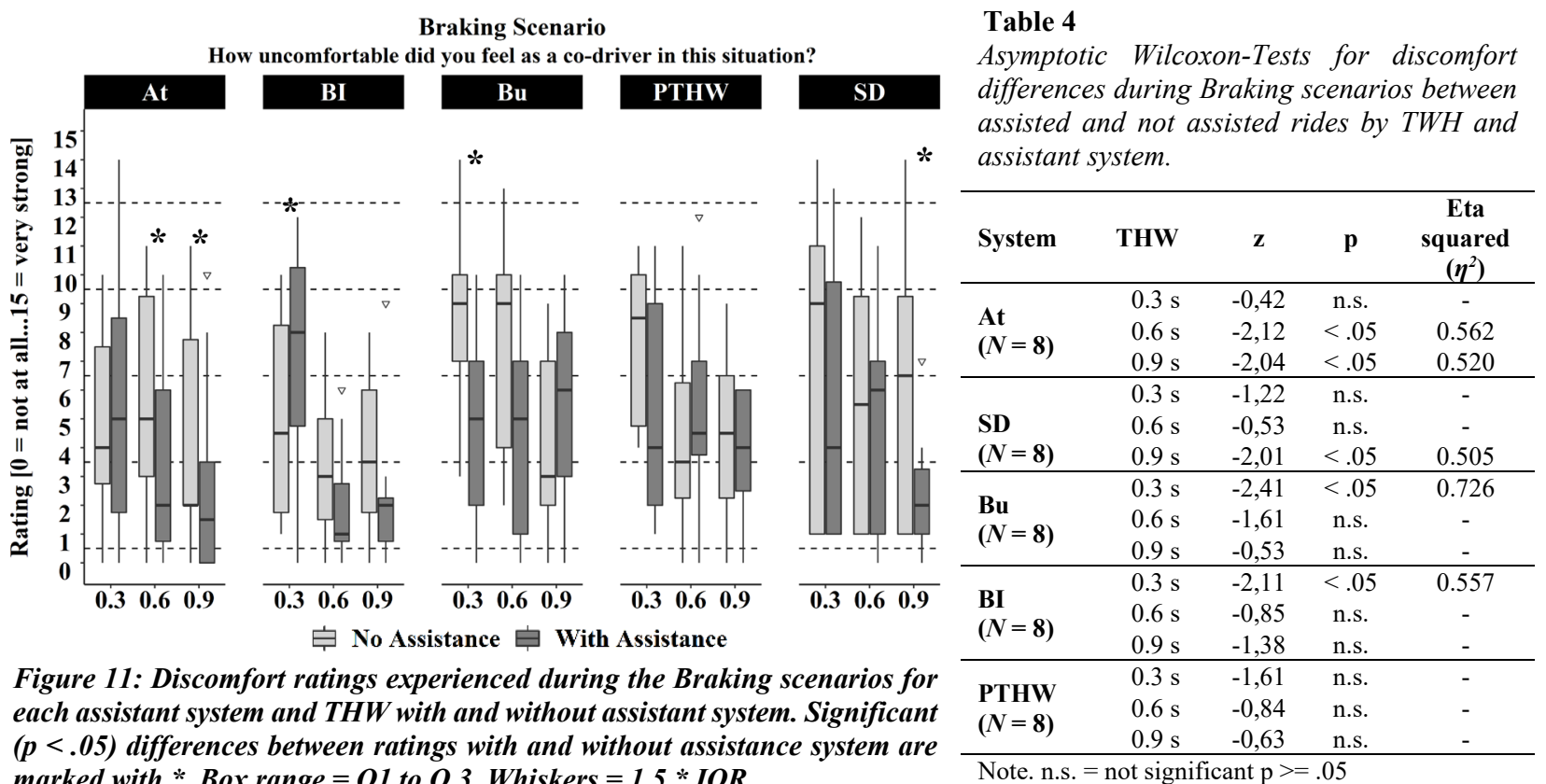

Figure 12 plots for each assistant system the experienced discomfort for the Following scenarios. Only the "Bu" assistant system reduced discomfort during short and medium THWs (Table 5). For all other assistant systems, no significant effect was found. Comparing the average discomfort ratings for the Braking and Following scenario without an assistant system, it appears that the participants experienced more discomfort during the Braking scenarios than during the Following scenarios. Additionally, the average discomfort ratings without an assistant system in the Following scenarios were already lower showing that the participants experienced only little or very little discomfort. 


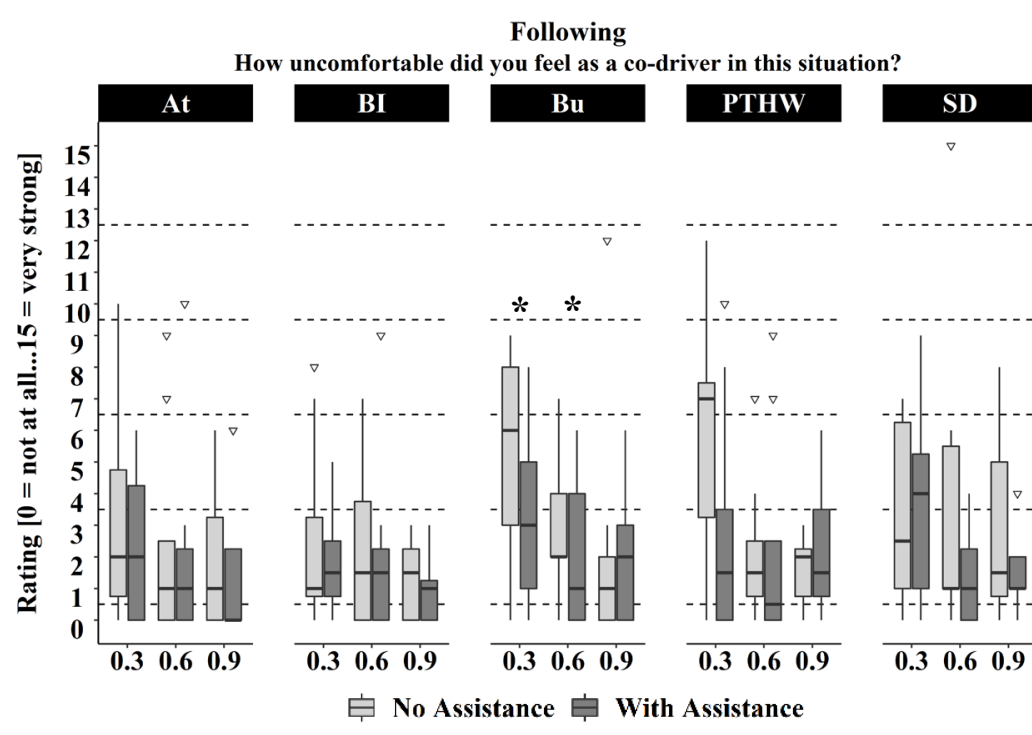

Figure 12: Discomfort ratings experienced during the Following scenarios for each assistant system and THW with assistant system and without. Significant $(p<.05)$ differences between ratings with and without assistance system are marked with *. Box range $=Q 1$ to $Q 3$. Whiskers $=1.5 * I Q R$.

When the "Bu" system was available participants felt significantly less exposed during short and medium distances in the Braking scenarios compared to runs without assistance system (Table 6). There was no reduction of their feeling of being exposed during the Following scenarios nor during Braking scenarios with larger THWs.

\subsubsection{Post inquiry}

The overall ratings in the post inquiry show that $75 \%$ of the participants (Figure 13 left) found the "At" assistance system very or extremely helpful to reduce their discomfort. Most of them argued that they felt more secure or had a positive feeling when knowing that a driver is focused.

Table 5

Asymptotic Wilcoxon-Tests for discomfort differences during Following scenarios between assisted and not assisted rides by THW and assistant system.

\begin{tabular}{lcccc}
\hline System & THW & $\mathbf{z}$ & $\mathbf{p}$ & $\begin{array}{c}\text { Eta } \\
\text { square } \\
\mathbf{d}\left(\boldsymbol{\eta}^{2}\right)\end{array}$ \\
\hline At & $0.3 \mathrm{~s}$ & $-0,85$ & n.s. & - \\
$(\boldsymbol{N}=\mathbf{8})$ & $0.6 \mathrm{~s}$ & $-0,14$ & n.s. & - \\
& $0.9 \mathrm{~s}$ & $-0,56$ & n.s. & - \\
\hline & $0.3 \mathrm{~s}$ & $-0,14$ & n.s. & - \\
SD & $0.6 \mathrm{~s}$ & $-1,83$ & n.s. & - \\
$(\boldsymbol{N}=\mathbf{8})$ & $0.9 \mathrm{~s}$ & $-1,29$ & n.s. & - \\
\hline Bu & $0.3 \mathrm{~s}$ & $-2,38$ & $<.05$ & 0.708 \\
$(\boldsymbol{N}=\mathbf{8})$ & $0.6 \mathrm{~s}$ & $-2,26$ & $<.05$ & 0.638 \\
\hline BI & $0.9 \mathrm{~s}$ & $-0,37$ & n.s. & - \\
$(\boldsymbol{N}=\mathbf{8})$ & $0.3 \mathrm{~s}$ & $-1,09$ & n.s. & - \\
\hline PTHW & $0.6 \mathrm{~s}$ & $-0,27$ & n.s. & - \\
$(\boldsymbol{N}=\mathbf{8})$ & $0.9 \mathrm{~s}$ & $-1,34$ & n.s. & - \\
\hline
\end{tabular}

Note. n.s. $=$ not significant $\mathrm{p}>=.05$
Table 6

Asymptotic Wilcoxon-Tests for differences of feeling exposed between "Bu" assisted and non-assisted rides by THW and scenario.

\begin{tabular}{lcccc}
\hline $\begin{array}{l}\text { Scenario } \\
(\boldsymbol{N}=\mathbf{8})\end{array}$ & THW & $\mathbf{z}$ & $\mathbf{p}$ & $\begin{array}{c}\text { Eta } \\
\mathbf{s q u a r e d} \\
\left(\boldsymbol{\eta}^{2}\right)\end{array}$ \\
\hline \multirow{4}{*}{ Following } & $0.3 \mathrm{~s}$ & $-1,61$ & n.s. & - \\
& $0.6 \mathrm{~s}$ & $-1,69$ & n.s. & - \\
& $0.9 \mathrm{~s}$ & $-0,94$ & n.s. & - \\
\hline \multirow{3}{*}{ Braking } & $0.3 \mathrm{~s}$ & $-2,37$ & $<.05$ & 0.702 \\
& $0.6 \mathrm{~s}$ & $-2,03$ & $<.05$ & 0.515 \\
& $0.9 \mathrm{~s}$ & $-0,11$ & n.s. & - \\
\hline
\end{tabular}

Note. n.s. $=$ not significant $\mathrm{p}>=.05$

(For more participant responses to this subjective question, see Table A.1 in the appendix). The "safety distance" system received such positive responses from $63 \%$ of the participants, who often reported that they could better assess the distance or situation. Additionally, $25 \%$ rated this system as medium helpful. $51 \%$ of the participants who experienced the "Bu", the "BI" or the "PTHW" assistant system rated it as very or extremely helpful in reducing their discomfort. The proportion of participants who reported the experienced assistant system as slightly or marginally helpful was highest for the "Bu" system with $38 \%$. Most stated the opinion that the system has no effect or could increase the anxiety of a passenger. There was only one participant who found the experienced co-driver assistant system ("BI") not helpful at all to reduce discomfort with the argument that information about the driving or braking process would not be relevant to a passenger.

The response distribution for the question of whether the participants would use the experienced assistant system showed similar ratios (Figure 13 right). The proportion of participants who answered that they would use the assistant system was highest for the "At" and the "PTHW" system with $75 \%$ and $63 \%$ followed by the systems "SD" and "BI". Additionally, $25 \%$ in the "SD" group said that it depends on the driver or situation whether they would use the system. In the "BI" group this amount was slightly lower with $13 \%$ because the other $13 \%$ of the participants were undecided. The lowest proportion was found in 
the "Bu" system group with $38 \%$ who would use the assistant system. $38 \%$ in this group said that it depends on the driver/situation whether they would use the system. The other $25 \%$ would not use the system because they did not see a need for it. They reported that instead, they would say something to the driver $(N=2)$. Additional responses to this question can be found in Table A.2 in the appendix.

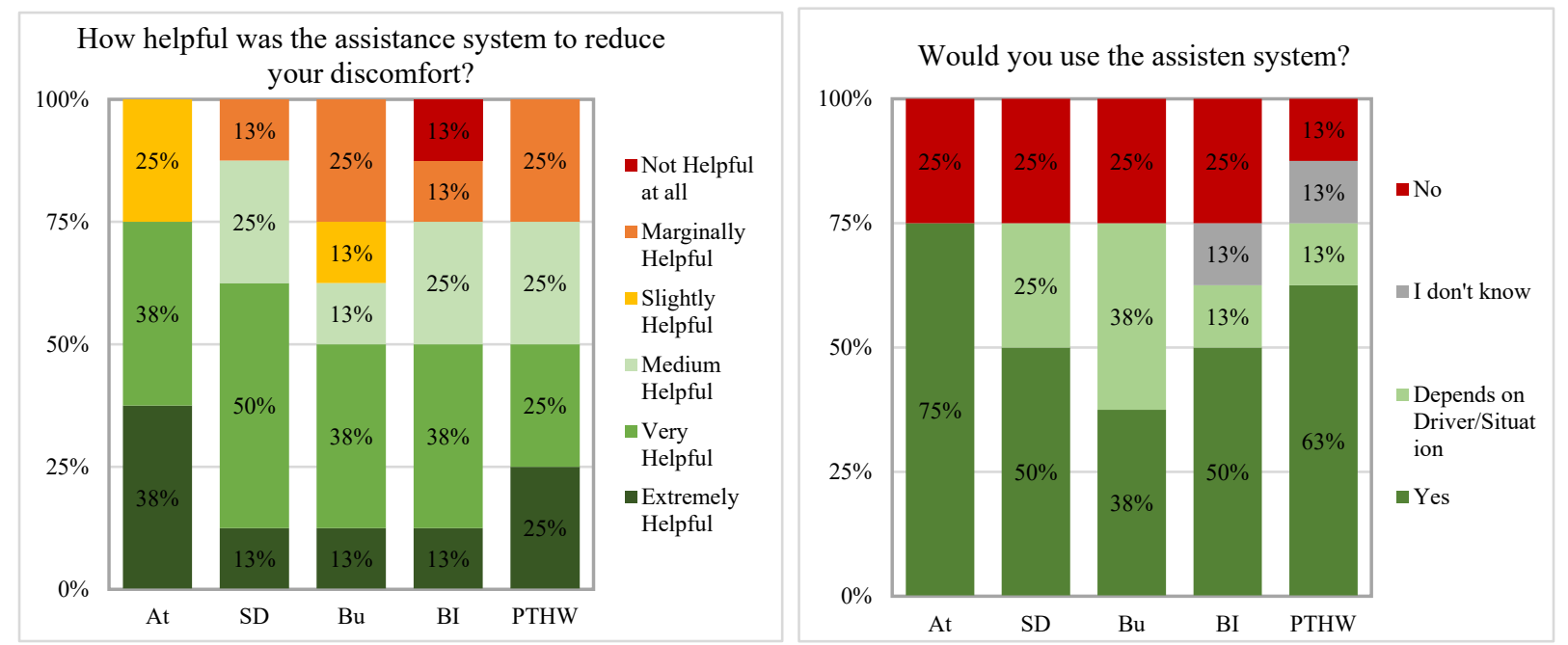

Figure 13: Left: Helpfulness ratings for the different assistance systems made in the post inquiry. Right: Reported intention to use the experienced assistant system.

\subsection{Discussion}

This section will discuss the results of the user study with respect to the hypotheses formulated in section 2 .

Table 7 shows a summary of this discussion. The general hypothesis 1 was that information about the cognitive state of the driver or a means for control can reduce passenger discomfort compared to rides with no information or control. The transparent information should lead to a better estimation of the situation or a validation of the own estimations in the situations which reduces passenger discomfort or prevents it. The provided control for the passenger should reduce their feeling of being exposed which consequently reduces their experienced discomfort. The main effect of the information-specific assistant systems and the controlspecific assistant system showed a significant reduction of passenger discomfort which confirms the main hypothesis.

Hypothesis H1.2 investigated if information about the attentiveness of the driver reduced passenger discomfort in comparison to no information about the attentiveness. The detailed investigations showed that hypothesis 1.2 concerning assistant system "At" can be accepted. The "At" system provided positive feedback about the attentiveness of the driver like distance regulation and the driver's ability for a reaction to sudden braking. The information about the attentiveness of the driver significantly reduced the experienced discomfort in the simulator study. However, this was only found for medium and large distances during the Braking scenario. It is possible that the short distances were too small for the participants to trust the system. This is supported by the significant relation between the helpfulness of the information displayed by the assistant system and the discomfort and criticality ratings in the situations. The lower the participants rated the helpfulness of the assistant system in the experienced situations, the lower was their trust in the driver and the higher was the rated criticality and their experienced discomfort (H1.1). In the Following scenarios, the system showed no reduction. This could be due to the fact that the discomfort for all THWs during the scenarios in the baseline was already very low. These very low discomfort ratings imply that the participants did not need an assistant system in these situations. Interestingly, in the feasibility study, the ratings for the Following scenarios were much higher even though the THWs were larger. This could be caused by higher acceleration rates in the feasibility study which could have led to the impression of a more "aggressive" driving style. 
Hypothesis H1.3 was concerned with the passenger discomfort reducing effect of the driver's experience based reference value in comparison to no information about it. The assistant system "PTHW" communicated this reference value which (s)he used to evaluate following distances to the passenger for a more accurate estimation of a situation. The results for the "PTHW" assistant system are similar to the "BI" assistant, which means that the system did not reduce passenger discomfort. Hypothesis 1.3 can therefore also be rejected. These results do not correspond to the findings by Khastgir et al. (2018) which showed that information about system capabilities increased driver trust, regardless of whether the capabilities were high or low. One reason named in the post inquiry by the participants was the subjective character of the "PTHW" which means that it does not automatically correspond to a safe distance. Another reason could be that the relevance of the information provided by the "PTHW" depends on the familiarity of the driver. The "PTHW" assistant system is more informative when the driver is unfamiliar to the passenger like a taxi or lift driver.

Hypothesis H1.4 examined the discomfort reducing effect of explicit information about the safety threshold provided to the passenger and driver in comparison to no information. The "SD" assistant system had the target to communicate to the passenger that both, the driver and the passenger, have a shared understanding of a "safe" distance to the front vehicle. The helpfulness of the "SD" information correlated positively with situation estimation. However, there was no relationship to trust or the criticality estimation (H1.1). The assistant system only showed a discomfort reduction during long THWs in the Braking scenarios. This means that the positive effect of transparent information indicated by the results of the studies by Chang et al. (2019), Yang et al. (2018) was only partly found for this system variation. However, in the post inquiry more than half of the participants rated the assistant system as very or extremely helpful in reducing their discomfort. This leads to the conclusion that hypothesis 1.4 can be only partially accepted.

In hypothesis H1.5 it was investigated if information about the braking intentions of the driver reduce passenger discomfort in comparison to no information about it. For this assistant system "BI", hypothesis 1.5 must be rejected. The system displayed information about driver actions that are relevant for the regulation of longitudinal velocity. This should reduce uncertainties on the part of the passenger regarding already executed actions or intentions of the driver. The results showed no reduction of passenger discomfort in all scenarios and even increased their discomfort during short THWs in the Braking scenarios. There was also no positive effect of braking information on trust in the driver. It is possible that the displayed braking process of the driver was perceived rather like a warning or a highlighting of criticality in the situation increasing their discomfort. Another explanation could be that the braking information was displayed too late in the situation to have the same positive effect as the more predictive information about the intentions of an automated vehicle like in the study by Chang et al. (2019) or Löcken et al. (2016). The information displayed by the system was also rated as helpful in fewer situations compared to the other systems.

The last hypothesis H1.6 examined if passenger discomfort is reduced by having influence as a passenger on the safety distance compared to no influence. The "Bu" system provided means of control and influence on the distance to the passenger. This should make it easier for passengers to communicate to the driver that the distance is too small for them. In contrast to the other systems, the "Bu" system also showed a reduction during short distances and in the Following scenarios. In some of the Braking scenarios, the system also led to a reduction of the participant's feeling of being exposed. This is also in line with the positive influence of control during automated driving found by Frison et al. (2017). Therefore, hypothesis 1.6 can be accepted. Despite a more positive effect on passenger discomfort than for example the "At" system, it was rated as less helpful by more participants in the post inquiry compared to the "At" system and showed the lowest number of participants who would use the system. This could be explained by the fact that some participants argued that they do not need such a system because they would say something to the driver or would prefer an automatic system. For the "Bu" system the relations showed that the higher the trust in the driver was rated, the lower the helpfulness of the provided control and the discomfort was (H1.1). This indicates that the passengers did not need the assistant system in situations in which they trusted the driver to handle it. 
The positive relation of helpfulness and discomfort as well as the negative relation between helpfulness and trust in the driver imply this. The more helpful the possibility to signal something to the driver (need for control) was rated, the higher the discomfort and the lower the trust in the driver was in these situations.

All in all, the presented information about the cognitive state of the driver seems mostly helpful for passengers during medium and longer THWs. The button system was also helpful during medium THWs but even more for short THWs, which is in line with the theory. The Information aims to help passengers to verify their estimations and prevent discomfort, while the button system should be a possibility to intervene when a situation already made them feel uncomfortable, which is especially the case during the short and medium THWs Since the short THWs were very short, it is natural and justified to feel uncomfortable in these situations. Therefore it makes sense that the test persons did not let themselves calm down only by presented information but only found an intervention helpful. The online ratings and the post inquiry ratings showed differences in the evaluation of the assistant systems. Except for the "Bu" system, the assistant systems were rated more helpful in reducing discomfort in the post inquiry than their discomfort reducing effect in the situations implied. This could be caused by the low discomfort ratings in the following scenarios even without an assistant system which reduced the number of situations in which the participants needed the information. But for the situations in which they felt discomfort, the systems were experienced as helpful.

While the systems are designed to address one point of the passenger discomfort model at a time to reduce discomfort, it is also conceivable to combine the systems. For example, it makes sense to combine the SD and $\mathrm{Bu}$ systems, as they complement each other. If the objectively indicated safety distance is not sufficient for the passenger in a certain situation, the passenger could simply signal this to the driver with the $\mathrm{Bu}$ system.

\section{Table 7}

Summarized results with the investigated topics in the left column and in the right column a ranking from best to worst rated assistant system.

\begin{tabular}{ll} 
Topics & Ranking \\
\hline Helpfulness of information/influence & At $>$ SD, Bu > BI, PTHW \\
\hline Helpfulness of assistant system to estimate situations better & At $>$ SD $>$ Bu $>$ BI, PTHW \\
\hline Relations to criticality estimation, discomfort, trust and feeling of being exposed & At $>$ Bu $>$ SD, BI, PTHW \\
\hline Reduction of discomfort & Bu > At $>$ SD $>$ PTHW > BI \\
\hline Reduction of feeling of being exposed (only investigated for Bu) & $\begin{array}{l}\text { Significant reductions only for short and medium } \\
\text { distance during Braking scenarios }\end{array}$ \\
\hline Post inquiry helpfulness ratings & At $>$ SD $>$ Bu, BI, PTHW \\
\hline Post inquiry assistant system use ratings & At $>$ PTHW $>$ SD, Bu $>$ BI \\
\hline
\end{tabular}

As mentioned in the introduction of the feasibility study, simulator studies, as well as real driving studies, have their advantages and disadvantages. Besides the higher safety and controllability in simulator studies, they have the disadvantage of being less realistic. The simulator used in this study was a static simulator without available longitudinal forces, which likely reduced the perceived criticality of the situations. The aim of future studies could be therefore to examine the influence of the tested co-driver assistance systems on discomfort under real driving conditions. Another limitation was the between design and the resulting low number of participants per assistant system. This probably reduced the possibility to find an effect of the assistant systems on other levels of the factors. A validation of the results in a within design with more participants can be also part of future studies.

\subsection{Conclusion and Future Research}

The simulator study showed that the proposed concepts to provide information about the cognitive state of the driver or the possibility to have more influence can have a positive effect on passenger discomfort. In the final evaluation, passengers found the provided information or influence helpful and showed interest in 
using the assistant systems. These results thus indicate that the transparent display of information from the "black box" driver can also have a positive effect on the passenger's driving experience. The same applies to the possibility of being able to intervene more as a passenger. A combination of these two investigated mechanisms in one passenger assistant system could be even more beneficial in terms of their discomfort. Assistant systems can therefore improve the driving experience and comfort not only for drivers but also for passengers. However, some further aspects need to be considered when developing passenger assistance systems.

An aspect that could not be investigated in this study for methodical reasons is the influence of the assistance systems on the acceptance of the driver and on the relationship between driver and passenger. It is possible, for example, that conflicts no longer arise because the passenger subjectively assesses situations more critically than the driver. Instead, conflicts may now arise because the driver does not keep the safety distance suggested by the SD system, or does not react to the Bu signal from the passenger. It could be possible that ignoring the safety relevant information made for example by the "SD" system could even increase passenger discomfort in comparison to the same situations experienced without such a system. Other negative effects of the assistant systems could be that the passenger distrusts the displayed system states, or the information might be displayed too late. If the information is only available to the front passenger, as is the case with the At system, it could also be that the driver feels controlled by the passenger. These possible side effects on the driver and the relationship between the two vehicle occupants can be part of further research. Additionally, the study was conducted with a driver unfamiliar to the participant. Since interviews (Ittner et al., 2020) showed that passengers more frequently travel with known drivers it would be another interesting topic to investigate the effect of the assistant systems under these conditions.

The different assistant system concepts presented in this work were mainly developed for the two most frequently named reasons for passenger discomfort in previous interviews and questionnaires (Ittner et al., 2020): close following and fast driving. This means that except for the general "At" system, whose information could be also helpful during other situations, the other ones presented information that is relevant mostly during close following on the autobahn. However, it is also possible to expand the cognitive co-driver discomfort model to other situations in which the driver regulates the driving task such as overtaking maneuvers or when the driver is distracted. Therefore, it is also possible to derive other HMI variations based on the co-driver discomfort model presented in section 2 providing different types of information about the cognitive state of the driver or providing other forms of influence. Investigating what influence other types of information may have on passenger discomfort could be part of future studies.

In conclusion, this work could show that it is possible to make a co-driver assistant system that reduces passenger discomfort. It also becomes clear that there is a lot of potential in taking the passenger more into account during the design process of assistance systems. Even rudimentary information, some of which is currently only displayed to the driver (e.g. drowsiness warning, distance indication with ACC), can also have positive effects on the passenger's driving experience if it is also available to them. This is not only relevant for the further development of assistant systems in conventional vehicles but also with higher automation levels, when the driver will start to become a passenger of the vehicle. So, there are more possibilities and needs to increase the comfort of passengers beyond infotainment systems.

Author Contributions: S.I., D.M., T.W., M.V.: Conceptualization; S.I., D.M., T.W., M.V.: methodology; S.I.: software; S.I., D.M., T.W.: validation; S.I.: formal analysis; S.I.: investigation; S.I., T.W.: resources; S.I.: data curation; S.I.: writing-original draft preparation; D.M., T.W., M.V.: writing-review and editing; S.I.: visualization; S.I., T.W.: project administration. All authors have read and agreed to the published version of the manuscript.

Funding: This project is part of a research program funded and supported by the Honda Research Institute Europe $\mathrm{GmbH}$. 
Acknowledgments: We would like to thank the technical staff of the WIVW GmbH for the implementation of the assistance systems (hardware and software) and our designer for conceptualizing the assistant system icons. We want to thank Alexandra Neukum for administrative support of our experiment.

Conflicts of Interest: This study was conducted as part of a research program of the Honda Research Institute Europe $\mathrm{GmbH}$. Thomas Weisswange is employee of this company. He contributed to the design of the study, review and editing of the manuscript and the decision to publish the results. 


\section{References}

Adell, E., Várhelyi, A., \& Bruel, M. D. F. L. (2008). Test of HMI alternatives for driver support to keep safe speed and safe distance-A simulator study. The Open Transportation Journal, 2(1). DOI: $\underline{10.2174 / 1874447800802010053}$

Aydogdu S., Seidler C., Schick B. (2019) Trust Is Good, Control Is Better? - The Influence of Head-Up Display on Customer Experience of Automated Lateral Vehicle Control. In: Krömker H. (eds) HCI in Mobility, Transport, and Automotive Systems. HCII 2019. Lecture Notes in Computer Science, vol 11596. Springer, Cham. https://doi.org/10.1007/978-3-030-22666-4 14

Bower, G. H., and Cohen, P. R. (1982). "Emotional influences in memory and thinking: data and theory," in Affect and Cognition, eds M. S. Clark and S. T. Fiske (Hillsdale, NJ: Erlbaum), 291-331

Carver, C. S., and Scheier, M. F. (2002). Control processes and self-organization as complementary principles underlying behavior. Pers. Soc. Psychol. Rev. 6, 304-315. DOI: 10.1207/S15327957PSPR0604_05

Chang, C. C., Grier, R. A., Maynard, J., Shutko, J., Blommer, M., Swaminathan, R., \& Curry, R. (2019). Using a situational awareness display to improve rider trust and comfort with an AV taxi. In Proceedings of the Human Factors and Ergonomics Society Annual Meeting (Vol. 63, No. 1, pp. 2083-2087). Sage CA: Los Angeles, CA: SAGE Publications. DOI:10.1177/1071181319631428

Charissis, V., Papanastasiou, S. (2010). Human-machine collaboration through vehicle head up display interface. Cogn Tech Work 12, 41-50. https://doi.org/10.1007/s10111-008-0117-0

Dadashi, N., Stedmon, A. W., \& Pridmore, T. P. (2013). Semi-automated CCTV surveillance: The effects of system confidence, system accuracy and task complexity on operator vigilance, reliance and workload. Applied Ergonomics, 44(5), 730-738. https://doi.org/10.1016/j.apergo.2012.04.012

Frison, A. K., Wintersberger, P., Riener, A., \& Schartmüller, C. (2017). Driving hotzenplotz: A hybrid interface for vehicle control aiming to maximize pleasure in highway driving. In Proceedings of the 9th international conference on automotive user interfaces and interactive vehicular applications (pp. 236244). https://doi.org/10.1145/3122986.3123016

Hesse, T., Schieben, A., Heesen, M., Dziennus, M., Griesche, S., \& Köster, F. (2013). Interaction design for automation initiated steering manoeuvres for collision avoidance. In 6. Tagung Fahrerassistenzsysteme. https://mediatum.ub.tum.de/doc/1187194/1187194.pdf

Hofauer, S., Michel, B., Weise, S., Karmann, A. J., Diermeyer, F., Stephan, A., ... \& Bendewald, L. (2018). HMI Strategy-Lateral and Longitudinal Control. In UR: BAN Human Factors in Traffic (pp. 105-118). Springer Vieweg, Wiesbaden. DOI 10.1007/978-3-658-15418-9_6

Hoff, K. A., \& Bashir, M. (2015). Trust in Automation: Integrating Empirical Evidence on Factors That Influence Trust. Human Factors: The Journal of the Human Factors and Ergonomics Society, 57(3), 407434. https://doi.org/10.1177/0018720814547570

Israel, B., Seitz, M., Bubb, H., \& Senner, B. (2010). Contact Analog Information in the Head-Up DisplayHow Much Information Supports the Driver? Advances in Ergonomics Modeling and Usability Evaluation, 163

Ittner, S., Mühlbacher, D., \& Weisswange, T. H. (2020). The discomfort of riding shotgun-Why many people don't like to be co-driver. Frontiers in Psychology, 11. https://doi.org/10.3389/fpsyg.2020.584309 
Kennedy, R. S., Lane, N. E., Berbaum, K. S., \& Lilienthal, M. G. (1993). Simulator sickness questionnaire: An enhanced method for quantifying simulator sickness. The international journal of aviation psychology, 3(3), 203-220. https://doi.org/10.1207/s15327108ijap0303_3

Khastgir, S., Birrell, S., Dhadyalla, G., \& Jennings, P. (2018). Calibrating trust through knowledge: Introducing the concept of informed safety for automation in vehicles. Transportation research part $C$ : emerging technologies, 96, 290-303. https://doi.org/10.1016/j.trc.2018.07.001

Lazarus, R. S. (1982). Thoughts on the relations between emotion and cognition. Am. Psychol. 37:1019. DOI: 10.1037/0003-066X.37.9.1019

Lazarus, R. S., and Folkman, S. (1984). Stress, Appraisal, and Coping. New York, NY: Springer.

Leventhal, H., and Scherer, K. (1987). The relationship of emotion to cognition: a functional approach to a semantic controversy. Cogn. Emot. 1, 3-28. DOI:10.1080/02699938708408361

Löcken, A., Heuten, W., \& Boll, S. (2016). AutoAmbiCar: using ambient light to inform drivers about intentions of their automated cars. In Adjunct Proceedings of the 8th International Conference on Automotive User Interfaces and Interactive Vehicular Applications (pp. 57-62). https://doi.org/10.1145/3004323.3004329

Maurer, B., Trösterer, S., Gärtner, M., Wuchse, M., Baumgartner, A., Meschtscherjakov, A., ... \& Tscheligi, M. (2014). Shared gaze in the car: towards a better driver-passenger collaboration. In Adjunct Proceedings of the 6th International Conference on Automotive User Interfaces and Interactive Vehicular Applications (pp. 1-6). https://doi.org/10.1145/2667239.2667274

Miller, G. A., Galanter, E., and Pribram, K. H. (1960). Plans and the Structure of Behavior. New York, NY: Holt. DOI: 10.1037/10039-000

Naujoks, F., Forster, Y, Wiedemann, K. and Neukum, A. (2017). A Human-Machine Interface for Cooperative Highly Automated Driving. Advances in Human Aspects of Transportation, 585-595. DOI: 10.1007/978-3-319-41682-3_49

Perterer, N., Meschtscherjakov, A., \& Tscheligi, M. (2015). Co-Navigator: an advanced navigation system for front-seat passengers. In Proceedings of the 7th International Conference on Automotive User Interfaces and Interactive Vehicular Applications (pp. 187-194). https://doi.org/10.1145/2799250.2799265

Ramzan, M., Khan, H. U., Awan, S. M., Ismail, A., Ilyas, M., \& Mahmood, A. (2019). A survey on stateof-the-art drowsiness detection techniques. IEEE Access, 7, 61904-61919. DOI: 10.1109/ACCESS.2019.2914373

Reymond, G., Kemeny, A., Droulez, J., \& Berthoz, A. (2001). Role of lateral acceleration in curve driving: Driver model and experiments on a real vehicle and a driving simulator. Human factors, 43(3), 483-495. https://doi.org/10.1518/001872001775898188

Rittger, L., \& Götze, M. (2018). HMI strategy-recommended action. In UR: BAN Human Factors in Traffic (pp. 119-150). Springer Vieweg, Wiesbaden. DOI: 10.1007/978-3-658-15418-9_7

Schieben, A., Griesche, S., Hesse, T., Fricke, N., \& Baumann, M. (2014). Evaluation of three different interaction designs for an automatic steering intervention. Transportation research part F: traffic psychology and behaviour, 27, 238-251. https://doi.org/10.1016/j.trf.2014.06.002 
Stevenson, A., and Lindberg, C. A. (2017). New Oxford American dictionary. Oxford: Oxford University Press

Trösterer, S., Wuchse, M., Döttlinger, C., Meschtscherjakov, A., \& Tscheligi, M. (2015). Light my way: Visualizing shared gaze in the car. In Proceedings of the 7th International Conference on Automotive User Interfaces and Interactive Vehicular Applications (pp. 196-203). https://doi.org/10.1145/2799250.2799258

Wierwille, W. W., Casali, J. G., \& Repa, B. S. (1983). Driver steering reaction time to abrupt-onset crosswinds, as measured in a moving-base driving simulator. Human factors, 25(1), 103-116. https://doi.org/10.1177/001872088302500110

Winkler, S., Powelleit, M., Kazazi, J., Vollrath, M., Krautter, W., Korthauer, A., ... \& Bendewald, L. (2018). HMI strategy-Warnings and interventions. In UR: BAN Human Factors in Traffic (pp. 75-103). Springer Vieweg, Wiesbaden. DOI 10.1007/978-3-658-15418-9_5

Winsum, W. V., \& Heino, A. (1996). Choice of time-headway in car-following and the role of time-tocollision information in braking. Ergonomics, 39(4), 579-592. DOI: 10.1080/00140139608964482

Yang, Y. , Karakaya, B., Dominioni, G. C., Kawabe, K., \& Bengler, K. (2018). An HMI Concept to Improve Driver's Visual Behavior and Situation Awareness in Automated Vehicle, 21st International Conference on Intelligent Transportation Systems (ITSC), Maui, HI, 2018, pp. 650-655, DOI: 10.1109/ITSC.2018.8569986 


\section{Appendix}

Table A.1

Positive and negative statements per assistant system made in the post inquiry as a reason for the chosen helpfulness rating

\begin{tabular}{|c|c|c|c|c|}
\hline & \multicolumn{2}{|l|}{ Positive Statements } & \multicolumn{2}{|l|}{ Negative Statements } \\
\hline & Reasons & $N=32$ & Reasons & $N=16$ \\
\hline \multirow[t]{2}{*}{ At } & $\begin{array}{l}\text { The system gives you security/a good } \\
\text { feeling when the driver is concentrated }\end{array}$ & 5 & Does not replace trust & 2 \\
\hline & & & Forces passenger to pay attention & 2 \\
\hline \multirow{4}{*}{ SD } & Better estimation of situation & 3 & No effect & 1 \\
\hline & The system gives you security & 3 & & \\
\hline & Certainty that nothing can happen & 1 & & \\
\hline & Support for the driver & 1 & & \\
\hline \multirow{4}{*}{ Bu } & Possibility to intervene & 3 & Negative effect/more anxiety & 2 \\
\hline & Discreet hint & 1 & Automatic system would be more effective & 1 \\
\hline & Driver behavior is confirmed & 1 & No help & 1 \\
\hline & Good for anxious passengers & 1 & & \\
\hline \multirow{4}{*}{ BI } & Certainty that everything in control & 2 & Irrelevant & 2 \\
\hline & Feedback of driver reaction & 2 & Negative effect/ more anxiety & 1 \\
\hline & $\begin{array}{l}\text { Only useful if you do not know the } \\
\text { driver }\end{array}$ & 1 & & \\
\hline & $\begin{array}{l}\text { Supportive, if passenger recognizes } \\
\text { situation possibly earlier }\end{array}$ & 1 & & \\
\hline \multirow{3}{*}{ PTHW } & Better estimation of (safety) distance & 4 & Senseless with preferred distance & 2 \\
\hline & More security/calming & 2 & Hard to switch off & 1 \\
\hline & Feeling less exposed & 1 & More useful for driver & 1 \\
\hline
\end{tabular}

Table A.2

Positive and negative reasons made in the post inquiry for the intention to use the experienced assistant system

\begin{tabular}{|c|c|c|c|c|}
\hline & \multicolumn{2}{|c|}{ Yes/Yes, but it depends on the driver or situation } & \multicolumn{2}{|l|}{ Maybe/I don't know/No } \\
\hline & Reasons & $N=33$ & Reasons & $N=\mathbf{1 0}$ \\
\hline \multirow{3}{*}{ At } & $\begin{array}{l}\text { Depends on situation (dichter Verkehr, } \\
\text { Nachtfahrt, Langstrecke) }\end{array}$ & 2 & No possibility of influence & 1 \\
\hline & When already built-in & 1 & & \\
\hline & The system provides security & 1 & & \\
\hline \multirow{4}{*}{ SD } & $\begin{array}{l}\text { Provides more information to better } \\
\text { assess the situation }\end{array}$ & 3 & $\begin{array}{l}\text { Negative effects like need would arise to } \\
\text { control the driver }\end{array}$ & 1 \\
\hline & When already built-in & 2 & $\begin{array}{l}\text { The more thinking is taken away, the less } \\
\text { you think for yourself. What if technology } \\
\text { fails? }\end{array}$ & 1 \\
\hline & Depends on driver/situation & 2 & & \\
\hline & More trust in driver & 1 & & \\
\hline \multirow{3}{*}{ Bu } & Depends on the driver/situation & 2 & $\begin{array}{l}\text { System actually not necessary, can also be } \\
\text { said }\end{array}$ & 2 \\
\hline & More comfort & 1 & & \\
\hline & Discreet influence is comfortable & 1 & & \\
\hline
\end{tabular}




\begin{tabular}{|c|c|c|c|c|}
\hline & $\begin{array}{l}\text { Support for the driver if you are } \\
\text { attentive as a passenger }\end{array}$ & \multicolumn{3}{|l|}{1} \\
\hline & $\begin{array}{l}\text { Would only use it if it is an automatic } \\
\text { system }\end{array}$ & \multicolumn{3}{|c|}{1} \\
\hline \multirow{5}{*}{ BI } & Depends on driver & 1 & No added value & 1 \\
\hline & Permanent use & 1 & Would not use it & 1 \\
\hline & Matter of habit & 1 & $\begin{array}{l}\text { I cannot judge because there was no } \\
\text { dangerous situation }\end{array}$ & 1 \\
\hline & Safety and security & 1 & & \\
\hline & $\begin{array}{l}\text { The system can give feedback when } \\
\text { something needs to be said }\end{array}$ & 1 & & \\
\hline \multirow{4}{*}{ PTHW } & If already installed & 4 & No effect & 1 \\
\hline & If you don't trust the driver & 2 & Possibly yes, but do not activate actively & 1 \\
\hline & Visual feedback & 2 & & \\
\hline & Depends on situation & 2 & & \\
\hline
\end{tabular}

Table A.3

Formulations of all investigated items

Criticality How safety critical was the situation?

Trust How much did you trust the driver in that situation?

Discomfort How uncomfortable did you feel as a passenger in the situation?

Estimate How helpful was the assistance system to better assess the situation?

Exposed How much did you feel exposed to the situation as a passenger? 